\title{
Investment timing and capacity choice in duopolistic competition under a jump-diffusion model
}

\author{
Xiaoqin $\mathrm{Wu}^{1} \cdot$ Zhijun $\mathrm{Hu}^{2}$
}

Received: 25 August 2020 / Accepted: 3 August 2021 / Published online: 21 August 2021

(c) The Author(s), under exclusive licence to Springer-Verlag GmbH Germany, part of Springer Nature 2021

\begin{abstract}
This paper aims to apply the real options game theoretic to study the impact of sudden events on the optimal investment timing and capacity choice in a duopoly market. We model the market demand and investment cost as the geometric Brownian motions with jumps driven by the Poisson processes. A new computing method independent on specific distribution functions is proposed for the real option models with jump processes of random frequency and amplitude. Based on this method, we find that both firms delay investment with a larger capacity as uncertainties of demand and investment cost increase. We also demonstrate that two firms both invest later and the optimal capacity relationship between them is ambiguous in the presence of two sources of uncertainty. Numerical simulation reveals that upward (downward) jump in demand and downward (upward) jump in investment cost cause the firms to invest earlier (later) with a larger (smaller) capacity. Finally, in a duopoly with symmetric firms, the first investor invests earlier than in an asymmetric duopoly due to the threat of preemption.
\end{abstract}

Keywords Duopoly market · Real option game · Jump-diffusion process · Investment decision

\section{Introduction}

Nowadays, uncertainty is a main characteristic of the business environment. When firms plan to introduce a new product or a new technology to the market, two common and difficult decisions are how much capacity to invest in and when to do it. The size of a firm's investment usually reflects the level of future production capacity of the firm, that is, the scale of project output formed through investment, which then affects the quality of the firm's investment. By investing with a large capacity, the firm takes a risk when demand and investment cost are

\footnotetext{
Zhijun $\mathrm{Hu}$

zjhu@gzu.edu.cn

Xiaoqin Wu

2414219294@qq.com

1 School of Mathematics and Statistics, Guizhou University, Guiyang 550025, China

2 School of Management, Guizhou University, Guiyang 550025, China
} 
uncertain. On the one hand, if the ex post demand is too low and investment cost is too large, the production capacity of the firm will be idle and the income may be too low to cover the investment cost. On the other hand, large-scale investment would enable the firm to capture a larger share of the market and make more money out of it if the realized demand is high and investment cost is low.

In the context of investment decisions under uncertainty, making "optimal" decisions may be a challenging task due to the unknown impacts of uncertainties and the strategic interactions between firms. The real world of investment is characterized by strategic competition among competitors, where each firm evaluates its own strategic competitiveness among the competing parties. Therefore, it is interesting to analyze how strategic interactions in a competitive environment affect both the optimal investment timing and the investment capacity levels under uncertain market conditions.

In this paper, we assume that there are two sources of uncertainty: market demand and investment cost. Thus, the firm needs to consider random fluctuations in income caused by uncertain demand and investment cost, and maximizes the value of firm by optimizing the investment timing and production capacity. Investment decision-making issues regarding these two sources of uncertainty can be found in many literatures, such as [1-4]. However, these studies only discuss how a single firm determines the optimal timing of an investment projects of a given size.

In recent two decades, the stream of research for real options has been extended to investments under competition by combining the game theoretic analysis with real options theory. The founding work on real options game theory was developed by Smets [5]. Dixit and Pindyck [1] proposed a continuous-time duopolistic real options game model that simplifies the original approach of Smets [5]. Nielsen [6] analyzed a duopoly stochastic entry game with both positive and negative externalities. Weeds [7] studied a real options game model with R\&D competition under a winner-takes-all patent system. Huisman and Kort [8] studied investment strategy in a duopoly market with future availability of new technology. Siddiqui and Takashima [9] developed a sequential two-stage game-theoretic real options model for capacity expansion investments under output price uncertainty. A detailed review of real options game-theoretic models can be found in Azevedo and Paxson [10].

Within a strategic real options framework, Huisman and Kort [11] firstly considered the investment decisions for both timing and capacity of two symmetric entrants in a new market and evaluated the impact of competition on social welfare. Lavrutich et al. [12] extended the duopoly model by considering the hidden competition of a third firm, and found that the follower of the two is more eager to invest in order to avoid being squeezed out of the market by the hidden competitor. Lavrutich [13] and Huberts et al. [14] also examined a duopoly investment game in which the firms choose the capacity and timing of their investment. In particular, Lavrutich [13] added an exit option to investigate entry and exit decisions under capacity sizing, Huberts et al. [14] assumed that one of the firms already has some capacity in place.

The existing real-options literature on capacity-timing games usually assumes the projected cash flow stream or product demand follows a geometric Brownian motion (GBM) process as in the Black and Scholes model, that is, the internal and external environment of a firm changes within a predictable range. The advantage of using the GBM is that it leads to a more simplified and closed-form solution which can be effectively analyzed for firm's investment decision. Besides, under the GBM framework, investment decision rules are clear and intuitive. However, a large body of empirical and theoretical studies already showed that the GBM is not rich enough to capture the patterns commonly found in most financial and commodity markets. For example, Mason [15] found that large sudden changes in the spot 
price of Henry Hub natural gas during the period from January 2004 to January 2010. Nunes and Pimentel [4] found that the number of worldwide sales of iPhones from 3rd quarter 2007 to 3 rd quarter 2016 do not fit GBM.

With the increasing complexity of current economic, social and environmental systems, unexpected sudden events such as financial crisis, plague (e.g., COVID-19 pandemic), and natural disasters (e.g., hurricane or earthquake), equipment and technology updates, institutional reform may lead to a sudden increase or decrease in demand and investment cost for certain products and cause major shifts in markets. Obviously, these shocks will greatly affect the investment decisions of firms. Considering that the involved processes may exhibit sample-path discontinuities, some real options literatures suggest using jump-diffusion process (e.g., $[4,16,17])$, in which discrete value changes are superimposed on the Brownian motion. As pointed out by [1], a GBM process with discrete jumps is more realistic to model the cash flow stream of a firm's project.

In the present study, we analyses the optimal investment decision of duopolistic firms under the two-factor jump-diffusion model with the first factor being the market demand and the second factor being the investment cost. The jumps may represent uncertainties caused by unexpected future events, including financial crisis, natural catastrophes, plague, technological innovation (e.g., [18]) and other sources. We develop a real option game model to examine the effects of sudden events on firms' optimal investment thresholds and optimal investment capacities.

In addition, most of the existing literatures assume that the amplitude of "jump" follows a specific probability distribution (e.g., [19-23]), and its limitation is that the analysis results will greatly depend on the assumption of this specific distribution. To avoid this limitation, we propose a new numerical method, which relaxes the assumption that the jump amplitude is constant or follows a specific distribution by introducing several simple statistical characteristic parameters to replace the complex and unmeasurable probability density function.

By numerical analysis, we obtain the following conclusions: First, the optimal investment thresholds of duopolistic firms under the two-factor diffusion model are larger than that of the Huisman \& Kort model [11], whereas the optimal capacities cannot be compared. Second, under the two-factor jump-diffusion model, the upward jumps in demand and downward jumps in investment cost have a positive impact on the optimal investment timing and capacity size of the firms, whereas the downward jumps in demand and upward jumps in investment cost have a negative impact. Finally, due to the threat of preemption, the two firms with symmetric costs invest earlier than those with asymmetric costs, and the first investor (leader) enters the market earlier with a larger capacity than the second investor (follower). Therefore, the jump-diffusion model can incorporate different scopes of investment uncertainty and increase the evaluation of investment opportunity.

The contribution of our paper can be seen from two angles. First, to the best of our knowledge, this is the first paper to consider the optimal investment problem of duopolistic firms with two sources of uncertainty. We extend the model considered by [11] to the jumpdiffusion uncertainty, in which the stochastic demand and investment cost of project are driven by jump-diffusion processes. Meanwhile, we extend the monopolistic firm's timing decision model of [4] to a duopolistic framework, and we further consider the firms' optimal capacity decision problem. Second, this paper proposes a new numerical solution method for the real option game model. An important improvement of this method is that under the framework of preserving the assumption of random distribution of "jump" amplitude, it provides a unified calculation rule for the valuation of real options with "jump", which does not depend on its specific probability distribution. Based on this method, the influence of sudden events on project investment is discussed. 
The remainder of this study is organized as follows. Section 2 describes the dynamics of stochastic processes involved. Section 3 introduces model valuation framework and discusses the monopoly investment decision problem. Section 4 studies the optimal investment timing and capacity decision in a duopoly market with two sources of uncertainty. Section 5 provides numerical analysis for the optimal investment decisions. We conclude in Sect. 6 and discuss some potential extensions. The proofs of all mathematical statements in the paper are provided in Appendix. The appendix contains all proofs.

\section{Basic setup and assumptions}

In this section, we mainly introduce the mathematical model and associated assumptions to derive the firm's investment decisions. We extend the approach used in Huisman and Kort [11] to deal with two sources of uncertainty and we assume both market demand and investment cost evolving according to jump-diffusion processes.

Generally speaking, stochastic process $S=\left\{S_{t}, t \geq 0\right\}$ is described by the jump-diffusion process, which includes the GBM (continuous process) and compound Poisson process (discrete process) as follows

$$
\frac{d S_{t}}{S_{t-}}=\mu d t+\sigma d B_{t}+d\left(\sum_{i=1}^{N_{t}} U_{i}\right), S_{0}=s_{0}>0
$$

where $s_{0}$ is the initial value of the process, $\mu$ is the drift coefficient, and $\sigma$ is the instantaneous volatility (excluding the impact of jumps). $\left\{B_{t}, t \geq 0\right\}$ is a standard Brownian motion on a filtered probability space $\left(\Omega, \mathcal{F},\left\{\mathcal{F}_{t}\right\}_{t \geq 0}, \mathbb{P}\right)$ satisfying the usual properties. $\left\{N_{t}, t \geq 0\right\}$ is a time-homogeneous Poisson process with intensity $\lambda$, and the value of $N_{t}$ represents the cumulative number of sudden events up to time $t$, that is, $N_{t}$ is the number of sudden changes in the stochastic process $S_{t}$ till time $t$. And thus, $d N=1$ with probability $\lambda d t$ and $d N=0$ with probability $1-\lambda d t$. The percentage of jumps are random variables, denoted by $\left\{U_{j}\right\}_{j \geq 1} \stackrel{i . i . d .}{\sim} U$, which means that though each jump is different, they are independent of each other and follow the same probability law. The notation $S_{t-}$ indicates that whenever there is a jump, the value of the process before the jump is considered. Furthermore, $\left\{U_{j}\right\}_{j \geq 1}$ is independent of the processes $d N_{t}$ and $d B_{t}$.

The Eq. (1) implies that $S_{t}$ follows a geometric Brownian motion, but over each time interval $d t$ there is a small probability that it will fall or go up to $\left(1+U_{j}\right)$ times as its original value, then it will continue fluctuating until another event occurs [1]. Note that, if the sudden event does not occur (i.e., either $\lambda=0$, or $U=0$, with probability one), then we obtain the standard GBM [8-11].

Following Merton [20], the solution of Equation (1) is given by

$$
S_{t}=s_{0} \exp \left[\left(\mu-\frac{\sigma^{2}}{2}\right) t+\sigma B_{t}\right] \prod_{i=1}^{N_{t}}\left(1+U_{i}\right)
$$

Considering the investment problem, we assume that there are two sources of uncertainty. More specifically, we assume that both the exogenous demand shock $\boldsymbol{X}=\left\{X_{t}, t \geq 0\right\}$ and unit investment cost (including the cost of operating per unit of production capacity) 
$\boldsymbol{I}=\left\{I_{t}, t \geq 0\right\}$ follow jump-diffusion processes

$$
\frac{d X_{t}}{X_{t-}}=\mu_{X} d t+\sigma_{X} d B_{t}^{X}+d\left(\sum_{i=1}^{N_{t}^{X}} U_{i}^{X}\right), X_{0}=x_{0}>0
$$

and

$$
\frac{d I_{t}}{I_{t-}}=\mu_{I} d t+\sigma_{I} d B_{t}^{I}+d\left(\sum_{i=1}^{N_{t}^{I}} U_{i}^{I}\right), I_{0}=i_{0}>0 .
$$

We distinguish the two processes by using index $X$ or $I$ for each parameter and assume that these processes are independent of each other, and the jump sizes of stochastic demand and unit investment cost are independent of each other. In particular, if there are no jumps in market demand and investment cost, i.e., $\lambda_{X}=\lambda_{I}=0$, then processes (3) and (4) are called two-factor diffusion processes. $U^{l}(l=X, I)$ take positive (negative) values indicates upward (downward) jumps. Nunes and Pimentel [4] assume that the jumps in demand process are negative and the jumps in investment cost are positive. However, Nunes et al. [18] assume that the cost of investment decreases with technology innovation by means of decrease jumps. Let $\mu_{1}$ and $\mu_{2}$ represent the expected jump amplitudes in stochastic demand and unit investment cost, respectively, i.e., $E\left(U^{X}\right)=\mu_{1}, E\left(U^{I}\right)=\mu_{2}$. Here, $\mu_{1}>0(<0)$ means an upward (downward) jump in demand, and $\mu_{2}>0(<0)$ means an upward (downward) jump in investment cost. Following [4,18], we consider the two scenarios: (1) $\mu_{1}>0, \mu_{2}<0$, and (2) $\mu_{1}<0, \mu_{2}>0$.

The risk-neutral discount rate is given by a constant $r$, and in order to guarantee convergence of firm value so that the option is exercised within a finite period of time (e.g., [17]), we make the following assumption.

Assumption $1 r-\mu_{X}-\lambda_{X} \mu_{1}>0$ and $r-\mu_{I}-\lambda_{I} \mu_{2}>0$.

\section{Benchmark case: monopoly market}

To better understand how the model works, we first discuss the benchmark case where a monopoly firm holds a single investment opportunity. Suppose a risk-neutral firm that is considering to undertake an irreversible investment to enter a market with uncertain demand and investment cost. The decision-making problem concerns both the investment timing and the capacity size of the production plant. When the current level of market demand is low and investment cost is high, the firm is idle, waiting for a better investment opportunity. Once the current levels of market demand and investment cost reach the optimal investment thresholds, it makes the investment, the production starts immediately and the firm becomes active on the market. If the firm invests in a plant with capacity $Q$, it will therefore have to pay total investment cost $I_{t} Q$. We assume that the output price at time $t$ is

$$
P_{t}=X_{t}\left(1-\eta Q_{t}\right)
$$

where $Q_{t}$ is total market output and $\eta>0$ is a fixed price sensitivity parameter. Note that in the multiplicative inverse demand function, if $Q_{t} \leq 1 / \eta$ holds, then the market is bounded above and it corresponds to a market with a limited number of potential customers. Following [11] and [13], we assume that after investing the firm always operates at full capacity. 


\subsection{Investment timing and capacity decisions}

The expected value of the firm can be stated as follows

$$
J\left(x_{0}, i_{0}\right)=\mathbb{E}_{\left(x_{0}, i_{0}\right)}\left[\int_{\tau}^{\infty} e^{-r t}\left(X_{t} Q(1-\eta Q)-I_{t} Q\right) d t\right]
$$

which indicates the value of the option when the firm executes it at time $\tau<+\infty$. Following [24], we refer to $J$ as the performance criterion. Moreover, $\mathbb{E}_{\left(x_{0}, i_{0}\right)}$ denotes the expectation operator conditional on the available information at time 0 with $\left(X_{0}, I_{0}\right)=\left(x_{0}, i_{0}\right), \tau$ is the moment of investment, and $Q$ is the acquired capacity level or quantity at time $\tau$.

Since Brownian motion and Poisson processes are Markovian, it follows that both processes $X_{t}$ and $I_{t}$ are also Markovian. Using the strong Markov property, we can state and prove a result of the performance criterion.

Theorem 1 The performance criterion can be rewritten as

$$
J(x, i, Q)=\mathbb{E}_{(x, i)}\left[e^{-r \tau} G\left(X_{\tau}, I_{\tau}, Q\right)\right],
$$

with

$$
G(x, i, Q)=\frac{x Q(1-\eta Q)}{m_{X}}-\frac{i Q}{m_{I}}
$$

where

$$
m_{X}=r-\mu_{X}-\lambda_{X} \mu_{1}, m_{I}=r-\mu_{I}-\lambda_{I} \mu_{2} .
$$

The goal of the monopoly firm is to maximize performance criterion with respect to the investment timing and capacity level, i.e., the firm solves the following optimal stopping problem

$$
V(x, i)=\sup _{\tau \geq 0, Q \geq 0} J(x, i, Q)=J^{\tau^{*}}(x, i), \quad x, i \geq 0,
$$

where $\tau^{*}$ is called the optimal stopping time. According to Theorem 1, given the current levels of demand $x$, unit investment cost $i$ and capacity $Q$, the profit of the monopoly firm is $G(x, i, Q)=\frac{x Q(1-\eta Q)}{m_{X}}-\frac{i Q}{m_{I}}$.

Therefore, when the value of the monopoly firm, $V(x, i)$, is equal to the profit that the firm obtains by investing, $G(x, i, Q)$, the firm should choose to invest immediately. On the contrary, if $V(x, i)>G(x, i, Q)$, it is more profitable for the monopolist to delay its investment. Intuitively, low investment cost and high market demand will prompt monopolist to invest immediately, while high investment cost and low market demand make monopolist postpone investment.

Furthermore, by applying optimal stopping theory (see, [24]), we derive that $V(x, i)$ satisfies the following Hamilton-Jacobi-Bellman (HJB) equation:

$$
\begin{aligned}
& \frac{1}{2} \sigma_{X}^{2} x^{2} \frac{\partial^{2} V(x, i)}{\partial x^{2}}+\frac{1}{2} \sigma_{I}^{2} i^{2} \frac{\partial^{2} V(x, i)}{\partial i^{2}}+\mu_{X} x \frac{\partial V(x, i)}{\partial x}+\mu_{I} i \frac{\partial V(x, i)}{\partial i}+\lambda_{X} E \\
& {\left[V\left(\left(1+U^{X}\right) x, i\right)\right]+\lambda_{I} E\left[V\left(x,\left(1+U^{I}\right) i\right)\right]-\left(r+\lambda_{X}+\lambda_{I}\right) V(x, i)=0,}
\end{aligned}
$$

where $E\left[V\left(\left(1+U^{X}\right) x, i\right)\right]$ and $E\left[V\left(x,\left(1+U^{I}\right) i\right)\right]$ represent the expected value functions when the jumps occur in market demand $\left(U^{X}\right)$ and unit investment cost $\left(U^{I}\right)$. 
Additionally, $V(x, i)$ must satisfy the following boundary conditions:

$$
\begin{aligned}
& V\left(x^{*}, i^{*}\right)=\frac{x^{*} Q(1-\eta Q)}{m_{X}}-\frac{i^{*} Q}{m_{I}}, \\
& \left.\frac{\partial V(x, i)}{\partial x}\right|_{x=x^{*}, i=i^{*}}=\frac{Q(1-\eta Q)}{m_{X}}, \\
& \left.\frac{\partial V(x, i)}{\partial i}\right|_{x=x^{*}, i=i^{*}}=-\frac{Q}{m_{I}} .
\end{aligned}
$$

where $\left(x^{*}, i^{*}\right)$ denote the trigger values in terms of demand and unit investment cost at the optimal stopping time $\tau^{*}$.

Following [1], we can apply a variable transformation method to reduce the problem from two-dimensional to one-dimensional. To this end, we let

$$
G(x, i, Q)=i\left[\frac{x Q(1-\eta Q)}{i m_{X}}-\frac{Q}{m_{I}}\right]=i g(y, Q),
$$

and assume that

$$
V(x, i)=i v(x / i)=i v(y),
$$

where $y=x / i$ represents the demand-to-cost ratio, $v$ is a function to be determined. Substituting (11) into (9) and it can be expressed as follows

$$
\begin{gathered}
\frac{1}{2}\left(\sigma_{X}^{2}+\sigma_{I}^{2}\right) y^{2} v^{\prime \prime}(y)+\left(\mu_{X}-\mu_{I}\right) y v^{\prime}(y)+\lambda_{X} E\left[v\left(\left(1+U^{X}\right) y\right)\right]+ \\
\lambda_{I} E\left[\left(1+U^{I}\right) v\left(\left(1+U^{I}\right)^{-1} y\right)\right]-\left(r-\mu_{I}+\lambda_{X}+\lambda_{I}\right) v(y)=0 .
\end{gathered}
$$

In order to solve Eq. (12), we need to deal with the two terms $E\left[v\left(\left(1+U^{X}\right) y\right)\right]$ and $E\left[\left(1+U^{I}\right) v\left(\left(1+U^{I}\right)^{-1} y\right)\right]$. According to [25], we assume that $\lambda_{X}=\lambda_{I}=0$, then the general solution of Eq. (12) is of the form $v(y)=A y^{\beta}$. Therefore, we assume

$$
\begin{aligned}
& E\left[v\left(\left(1+U^{X}\right) y\right)\right]=E\left[\left(1+U^{X}\right)^{\beta}\right] v(y), \\
& E\left[\left(1+U^{I}\right) v\left(\left(1+U^{I}\right)^{-1} y\right)\right]=E\left[\left(1+U^{I}\right)^{1-\beta}\right] v(y) .
\end{aligned}
$$

Substituting (13) and (14) into (12) yields

$$
\begin{aligned}
& \frac{1}{2}\left(\sigma_{X}^{2}+\sigma_{I}^{2}\right) y^{2} v^{\prime \prime}(y)+\left(\mu_{X}-\mu_{I}\right) y v^{\prime}(y)+\lambda_{X} E\left[\left(1+U^{X}\right)^{\beta}\right] \\
& v(y)+\lambda_{I} E\left[\left(1+U^{I}\right)^{1-\beta}\right] v(y)-\left(r-\mu_{I}+\lambda_{X}+\lambda_{I}\right) v(y)=0 .
\end{aligned}
$$

To solve Eq. (15), we derive a new numerical integration method for arbitrary jump magnitudes $U^{X}$ and $U^{I}$ in the Eq. (15) and with sufficiently high accuracy. Following Westman and Hanson [26], we use the principle of Gauss-Statistics quadrature to replace the complex and unmeasurable probability density function of $U^{X}$ and $U^{I}$ with several statistical moments. For the continuous functions $\left(1+U^{X}\right)^{\beta}$ and $\left(1+U^{I}\right)^{1-\beta}$, if the probability density functions 
of $U^{X}$ and $U^{I}$ are $\varphi\left(y_{1}\right)$ and $\psi\left(y_{2}\right)$, respectively, then $E\left[\left(1+U^{X}\right)^{\beta}\right]$ and $E\left[\left(1+U^{I}\right)^{1-\beta}\right]$ can be expanded using a two-node approximation as follows

$$
\begin{aligned}
& E\left[\left(1+U^{X}\right)^{\beta}\right]=\int_{\mathcal{Y}_{1}}\left(1+y_{1}\right)^{\beta} \varphi\left(y_{1}\right) d y_{1} \simeq a_{1}\left(1+y_{11}\right)^{\beta}+a_{2}\left(1+y_{12}\right)^{\beta}, \\
& E\left[\left(1+U^{I}\right)^{1-\beta}\right]=\int_{\mathcal{Y}_{2}}\left(1+y_{2}\right)^{1-\beta} \psi\left(y_{2}\right) d y_{2} \simeq b_{1}\left(1+y_{21}\right)^{1-\beta}+b_{2}\left(1+y_{22}\right)^{1-\beta},
\end{aligned}
$$

where $a_{1}, a_{2}, b_{1}$ and $b_{2}$ are the weights, $y_{11}, y_{12} \in \mathcal{Y}_{1}$ and $y_{21}, y_{22} \in \mathcal{Y}_{2}$ are the nodes. After some algebra, these parameters can be expressed as

$$
\begin{aligned}
& a_{1}=\frac{1}{2}\left(1+S k_{1} / 2 \sqrt{\left(S k_{1} / 2\right)^{2}+1}\right), a_{2}=\frac{1}{2}\left(1-S k_{1} / 2 \sqrt{\left(S k_{1} / 2\right)^{2}+1}\right) \\
& y_{11}=\mu_{1}+\left(\frac{S k_{1}}{2}-\sqrt{\left(\frac{S k_{1}}{2}\right)^{2}+1}\right) \sigma_{1}, y_{12}=\mu_{1}+\left(\frac{S k_{1}}{2}+\sqrt{\left(\frac{S k_{1}}{2}\right)^{2}+1}\right) \sigma_{1}, \\
& b_{1}=\frac{1}{2}\left(1+S k_{2} / 2 \sqrt{\left(S k_{2} / 2\right)^{2}+1}\right), b_{2}=\frac{1}{2}\left(1-S k_{2} / 2 \sqrt{\left(S k_{2} / 2\right)^{2}+1}\right) \\
& y_{21}=\mu_{2}+\left(\frac{S k_{2}}{2}-\sqrt{\left(\frac{S k_{2}}{2}\right)^{2}+1}\right) \sigma_{2}, y_{22}=\mu_{2}+\left(\frac{S k_{2}}{2}+\sqrt{\left(\frac{S k_{2}}{2}\right)^{2}+1}\right) \sigma_{2},
\end{aligned}
$$

where $\mu_{1}, \mu_{2}$ are the expected values of $U^{X}$ and $U^{I}$ respectively, $\mu_{1}$ means average action strength of jump in demand, and $\mu_{2}$ means average action strength of jump in unit investment cost. Meanwhile, $\sigma_{1}$ and $\sigma_{2}$ are the standard deviations of $U^{X}$ and $U^{I}$ respectively, $\sigma_{1}$ represents the volatility of action strength of jump in demand, and $\sigma_{2}$ represents the volatility of action strength of jump in unit investment cost. Furthermore, $S k_{1}$ and $S k_{2}$ are, respectively, the skewnesses of $U^{X}$ and $U^{I}, S k_{1}$ implies the action direction of jump in demand, and $S k_{2}$ implies the action direction of jump in unit investment cost. If $S k_{1}>0\left(S k_{2}<0\right)$, it indicates that there is a higher probability that the demand (unit investment cost) jump will cause an abnormal increase in the project value; On the contrary, if $S k_{1}<0\left(S k_{2}>0\right)$, then there is a higher probability that the demand (unit investment cost) jump will cause an abnormal decrease in the project value.

Now, the weights and nodes are no longer depend on the the specific form of the distributions of $U^{X}$ and $U^{I}$, but only depend on three statistical moments: means, standard deviations, and skewnesses. Following [26], after some simple manipulations, Eq. (15) can be rewritten as

$$
\begin{aligned}
& \frac{1}{2}\left(\sigma_{X}^{2}+\sigma_{I}^{2}\right) y^{2} v^{\prime \prime}(y)+\left(\mu_{X}-\mu_{I}\right) y v^{\prime}(y)+\lambda_{X}\left[a_{1}\left(1+y_{11}\right)^{\beta}+a_{2}\left(1+y_{12}\right)^{\beta}\right] v(y) \\
& \quad+\lambda_{I}\left[b_{1}\left(1+y_{21}\right)^{1-\beta}+b_{2}\left(1+y_{22}\right)^{1-\beta}\right] v(y)-\left(r-\mu_{I}+\lambda_{X}+\lambda_{I}\right) v(y)=0 .
\end{aligned}
$$

Eq. (16) is the Cauchy-Euler equation, whose solution is $v(y)=A_{1} y^{\beta_{1}}+A_{2} y^{\beta_{2}}$, where $\beta_{1}$ and $\beta_{2}$ are, respectively, the positive and negative roots of the following equation

$$
\begin{gathered}
\frac{1}{2}\left(\sigma_{X}^{2}+\sigma_{I}^{2}\right) \beta(\beta-1)+\left(\mu_{X}-\mu_{I}\right) \beta+\lambda_{X}\left[a_{1}\left(1+y_{11}\right)^{\beta}+a_{2}\left(1+y_{12}\right)^{\beta}\right] \\
+\lambda_{I}\left[b_{1}\left(1+y_{21}\right)^{1-\beta}+b_{2}\left(1+y_{22}\right)^{1-\beta}\right]-\left(r-\mu_{I}+\lambda_{X}+\lambda_{I}\right)=0 .
\end{gathered}
$$

Since $\beta_{2}<0$, if $y \rightarrow 0$, then $y^{\beta_{2}} \rightarrow \infty$, which implies that $A_{2}=0$. Thus, the solution of Eq. (16) must take the form $v(y)=A_{1} y^{\beta_{1}}$. 
In this dynamic setting, the optimal investment decision is composed of two parts: timing and capacity. Let the trigger value $y^{*}=x^{*} / i^{*}$ denotes a point at which the monopolist is indifferent between choosing to invest immediately or delay investment, and $Q^{*}$ is the corresponding capacity level. Hence, we can describe the optimal investment timing as $\tau^{*}:=$ $\inf \left\{t>0 \mid y_{t} \geq y^{*}\right\}$. Namely, the optimal investment timing is equal to the moment when the exogenous demand-to-cost ratio $y_{t}=x_{t} / i_{t}$ first reaches $y^{*}$.

Combined with the boundary condition of Eq. (16), we can derive the optimal investment strategy and the value of firm as follows

Theorem 2 The value function of the monopoly firm is equal to

$$
v(y)= \begin{cases}A_{1} y^{\beta_{1}}, & y<y^{*} \\ \frac{\left(m_{I} y-m_{X}\right)^{2}}{4 y \eta m_{X} m_{I}^{2}}, & y \geq y^{*}\end{cases}
$$

where

$$
A_{1}=\frac{1}{\left(\beta_{1}^{2}-1\right) m_{I} \eta}\left[\frac{\left(\beta_{1}+1\right) m_{X}}{\left(\beta_{1}-1\right) m_{I}}\right]^{-\beta_{1}},
$$

and $\beta_{1}$ is the positive root of $E q$. (17).

The optimal investment threshold $y^{*}$ and the corresponding capacity level $Q^{*}$ are given by

$$
\begin{gathered}
y^{*}=\frac{\left(\beta_{1}+1\right) m_{X}}{\left(\beta_{1}-1\right) m_{I}}, \\
Q^{*}=\frac{1}{\left(\beta_{1}+1\right) \eta} .
\end{gathered}
$$

\subsection{Consumer surplus and social welfare}

To investigate the impacts of investment timing and size on social welfare, we first evaluate the consumer surplus. Following [11], given the demand shock $X$ and the capacity $Q$ of the firm, the instantaneous consumer surplus is equal to

$$
\int_{P(Q)}^{X} D(P) d P=\frac{1}{2} \eta X Q^{2}
$$

where $P(Q)=X(1-\eta Q)$ and $D(P)=\frac{1}{\eta}\left(1-\frac{P}{X}\right)$. The total expected consumer surplus (CS) is

$$
C S(x, Q)=E\left[\int_{t}^{\infty} \frac{1}{2} X_{s} Q^{2} \eta e^{-r(s-t)} d s \mid X_{t}=x\right]=\frac{x \eta Q^{2}}{2 m_{X}} .
$$

The expected producer surplus (PS) corresponds to the monopolist's value is

$$
P S(x, i, Q)=\frac{x Q(1-\eta Q)}{m_{X}}-\frac{i Q}{m_{I}} .
$$

Now, we can calculate the total expected social welfare (TS) as their sum:

$$
T S(x, i, Q)=C S(x, Q)+P S(x, i, Q)=\frac{x Q(2-\eta Q)}{2 m_{X}}-\frac{i Q}{m_{I}} .
$$


Let

$$
T S(x, i, Q)=i S W(y, Q)=i\left(\frac{y Q(2-\eta Q)}{2 m_{X}}-\frac{Q}{m_{I}}\right)
$$

Substituting (20) and (21) into (26), we can obtain that at the moment of investment, the expected social welfare is given by

$$
S W\left(y^{*}, Q^{*}\right)=\frac{3}{2\left(\beta_{1}+1\right)\left(\beta_{1}-1\right) \eta m_{I}} .
$$

On the other hand, the investment threshold and size that maximize social welfare in a monopoly market are

$$
\begin{gathered}
y_{W}^{*}=\frac{\left(\beta_{1}+1\right) m_{X}}{\left(\beta_{1}-1\right) m_{I}}=y^{*}, \\
Q_{W}^{*}=\frac{2}{\left(\beta_{1}+1\right) \eta}=2 Q^{*} .
\end{gathered}
$$

This result is the same as in Huisman and Kort [11], that is, the welfare-maximizing investment threshold is consistent with the optimal investment threshold of monopoly firm, while the welfare-maximizing capacity is twice that of the monopolist's optimal capacity.

In addition, the expected social welfare with a welfare-maximizing policy at the moment of investment is given by

$$
S W\left(y_{W}^{*}, Q_{W}^{*}\right)=\frac{2}{\left(\beta_{1}+1\right)\left(\beta_{1}-1\right) m_{I} \eta} .
$$

Therefore, in a monopoly market, the loss of welfare at the moment of investment equals

$$
S W_{L} \equiv S W\left(y_{W}^{*}, Q_{W}^{*}\right)-S W\left(y^{*}, Q^{*}\right)=\frac{1}{2\left(\beta_{1}+1\right)\left(\beta_{1}-1\right) m_{I} \eta} .
$$

\section{Duopoly market}

Now, we investigate a duopoly market in which two firms compete with homogeneous goods or serve a particular demand. Both firms are assumed to be risk neutral and profit maximizers with constant time discount rate $r$. Further, the two firms have the option to wait for their optimal timing to enter the market. Similar to literature $[11,27,28]$, we call the firm that enters the market first as the leader, and the other as the follower. Denote by $Q_{L}$ and $Q_{F}$ the investment sizes of the leader and the follower, respectively. When both firms are active in the market, the total market output is equal to $Q=Q_{L}+Q_{F}$. Following [11-13], we assume that the firms invest that always produce at full capacity and sell all their products to the market.

To provide as much intuition as possible, we analyze two situations in which two firms face the same or different investment costs. Several causes can lead to a situation that investment costs are heterogeneous among firms. For instance, the firms may have different accesses to capital markets, or show different degrees of organizational flexibility in implementing a new production technology [29]. The two firms face a multiplicative demand curve as specified in Eq. (5). Following the standard procedure in real option games, we analyze the problem backwards in time. 


\subsection{Asymmetric costs}

We first consider a situation in which the investment cost is strongly asymmetric. Without loss of generality, we make the following assumption about the unit investment costs $I_{1 t}, I_{2 t}$ $(t \geq 0)$ of two firms.

Assumption $2 I_{1 t}=I_{t}<\kappa I_{t}=I_{2 t}$, where $\kappa>1$.

where the fixed unit investment cost parameters of firm 1 and 2 are 1 and $\kappa$, respectively, and the process $I_{t}$ satisfy Eq. (4).

Assumption 2 indicates that the unit investment costs of both firms follow the same dynamic, and firm 1 has a significant cost advantage over firm 2 . Therefore, we assume firm 1 is the leader and firm 2 is the follower in the following analysis.

Denote by $y_{F}=x_{F} / i$ and $y_{L}=x_{L} / i$ the investment timing of the leader and follower, respectively. It is assumed that the leader investment has already taken place when the follower makes investment decision, therefore the follower cannot influence the investment decision of the leader. For a given level of the leader's investment capacity $Q_{L}$, the optimal investment timing $y_{F}^{*}$ and the investment capacity $Q_{F}^{*}$ of the follower are functions of $Q_{L}$. The optimal investment threshold and capacity level of the follower are described in the following theorem.

Theorem 3 Given the current level $y=x / i$ and the capacity $Q_{L}$ of the leader, the optimal capacity level $Q_{F}^{*}\left(y, Q_{L}\right)$ of the follower is equal to

$$
Q_{F}^{*}\left(y, Q_{L}\right)=\frac{1}{2 \eta}\left(1-\eta Q_{L}-\frac{\kappa m_{X}}{y m_{I}}\right) .
$$

The follower's value function $v_{F}^{*}\left(y, Q_{L}\right)$ is

$$
v_{F}^{*}\left(y, Q_{L}\right)= \begin{cases}A_{F}\left(Q_{L}\right) y^{\beta_{1}}, & y<y_{F}^{*}\left(Q_{L}\right) \\ \frac{\left[y m_{I}\left(1-\eta Q_{L}\right)-\kappa m_{X}\right]^{2}}{4 y \eta m_{X}}, & y \geq y_{F}^{*}\left(Q_{L}\right),\end{cases}
$$

where

$$
\begin{aligned}
& A_{F}\left(Q_{L}\right)=\frac{1-\eta Q_{L}}{\left(\beta_{1}^{2}-1\right) m_{I} \eta}\left[\frac{\left(\beta_{1}-1\right)\left(1-\eta Q_{L}\right) m_{I}}{\left(\beta_{1}+1\right) \kappa m_{X}}\right]^{\beta_{1}}, \\
& y_{F}^{*}\left(Q_{L}\right)=\frac{\left(\beta_{1}+1\right) \kappa m_{X}}{\left(\beta_{1}-1\right)\left(1-\eta Q_{L}\right) m_{I}},
\end{aligned}
$$

accordingly

$$
Q_{F}^{*}\left(Q_{L}\right) \equiv Q_{F}^{*}\left(y_{F}^{*}\left(Q_{L}\right), Q_{L}\right)=\frac{1-\eta Q_{L}}{\left(\beta_{1}+1\right) \eta} .
$$

Compared with Theorem 2, the results of Theorem 3 show that the factor $1-\eta Q_{L}$ appears in $y_{F}^{*}\left(Q_{L}\right)$ and in $Q_{F}^{*}\left(Q_{L}\right)$. This is because the leader has already invested in capacity, which means that, according to equation (5), when $Q_{F}=0$, the maximal output price is reduced by the factor $\eta Q_{L}$.

In the next step we analyze the leader's investment decision. Similar to [11], the leading firm can adopt two possible strategies: entry deterrence or entry accommodation. Entry deterrence corresponds to sequential investment and enables the leading firm to gain a monopoly profit for a period of time starting from its investment until the follower enters. On the contrary, entry accommodation leads to an immediate investment of the follower, that is, the 
follower invests at the same time as the leader. The leader can use its optimal capacity $Q_{L}$ as a tool to enforce either one of these two strategies. According to Eq. (35), it can be seen that the deterrence strategy occurs when the leading firm invests in a capacity size $Q_{L}$ that is larger than $\widehat{Q_{L}}(y)$, such that

$$
\widehat{Q_{L}}(y)=\frac{1}{\eta}\left(1-\frac{\left(\beta_{1}+1\right) \kappa m_{X}}{\left(\beta_{1}-1\right) y m_{I}}\right) .
$$

Therefore, in the opposite case, that is, $Q_{L} \leq \widehat{Q_{L}}(y)$, the leader and the follower invest simultaneously.

Notice that $y_{F}^{*}\left(Q_{L}\right)$ is increasing in $Q_{L}$ (see Eq. (35)), this means that the leading firm can expand its monopoly period by investing in a lager capacity. According to Eq. (36), another motivation for the leader to invest in a large capacity is that the capacity $Q_{F}^{*}\left(Q_{L}\right)$ decreases with $Q_{L}$.

We first discuss the leader's deterrence strategy. The leader's value function with an entry deterrence strategy is given by

$$
g_{L}^{\text {det }}\left(y, Q_{L}\right)=\frac{y\left(1-\eta Q_{L}\right) Q_{L}}{m_{X}}-\frac{Q_{L}}{m_{I}}-\left(\frac{y}{y_{F}^{*}\left(Q_{L}\right)}\right)^{\beta_{1}} \frac{y_{F}^{*}\left(Q_{L}\right) \eta Q_{F}^{*}\left(Q_{L}\right) Q_{L}}{m_{X}} .
$$

Since the leader uses the entry deterrence strategy, it generates monopoly profits for a certain period of time, given by the first item of the value function. The second item represents the investment cost required to install capacity with a quantity of $Q_{L}$. Moreover, as the leader cannot be in a monopoly position all the time, the follower will enter the market at some point in time, which decreases the profit of the leader. Therefore, we make a negative correction to the value function of leader by subtracting the third term, where $\left(y / y_{F}^{*}\left(Q_{L}\right)\right)^{\beta_{1}}$ is the stochastic discount factor that discounted from the moment of the follower's investment $y_{F}^{*}\left(Q_{L}\right)$ back to $y$.

The following Theorem 4 summarizes the leader's optimal investment decision when it uses the entry deterrence policy. Substituting (35) and (36) into (38) gives (41) in Theorem 4.

Theorem 4 The leader will consider the deterrence strategy whenever the current level of demand-to-cost ratio y lies within the interval $\left(y_{1}^{\text {det }}, y_{2}^{\text {det }}\right)$, where $y_{1}^{\text {det }}$ is the positive root of the following nonlinear equation

$$
\frac{y_{1}^{\text {det }}}{m_{X}}-\frac{1}{m_{I}}-\left[\frac{y_{1}^{\text {det }}\left(\beta_{1}-1\right) m_{I}}{\left(\beta_{1}+1\right) \kappa m_{X}}\right]^{\beta_{1}} \frac{\kappa}{\left(\beta_{1}-1\right) m_{I}}=0,
$$

and

$$
y_{2}^{\text {det }}=\frac{\left(\beta_{1}+1\right) m_{X}}{\left(\beta_{1}-1\right) m_{I}}\left[\beta_{1}(\kappa-1)+\kappa+1\right] .
$$

Given that the leader invests at $y$, the value function of the leader is as follows

$$
\begin{aligned}
g_{L}^{\text {det }}(y)= & \frac{y\left(1-\eta Q_{L}^{\text {det }}(y)\right) Q_{L}^{\text {det }}(y)}{m_{X}}-\frac{Q_{L}^{\text {det }}(y)}{m_{I}} \\
& -\left[\frac{y\left(\beta_{1}-1\right)\left(1-\eta Q_{L}^{\text {det }}(y)\right) m_{I}}{\left(\beta_{1}+1\right) \kappa m_{X}}\right]^{\beta_{1}} \frac{\kappa Q_{L}^{\text {det }}(y)}{\left(\beta_{1}-1\right) m_{I}},
\end{aligned}
$$


and the optimal investment capacity $Q_{L}^{\text {det }}(y)$ is implicitly determined by

$$
\begin{aligned}
& \frac{y\left(1-2 \eta Q_{L}^{\text {det }}\right)}{m_{X}}-\frac{1}{m_{I}}-\left[\frac{y\left(\beta_{1}-1\right)\left(1-\eta Q_{L}^{\text {det }}\right) m_{I}}{\left(\beta_{1}+1\right) \kappa m_{X}}\right]^{\beta_{1}} \\
& \frac{\kappa\left(1-\left(\beta_{1}+1\right) \eta Q_{L}^{\text {det }}\right)}{\left(\beta_{1}-1\right)\left(1-\eta Q_{L}^{\text {det }}\right) m_{I}}=0 .
\end{aligned}
$$

Furthermore, given that $y<y_{L}^{\text {det }}$, the optimal investment threshold $y_{L}^{\text {det }}$ and the corresponding capacity $Q_{L}^{\text {det }}$ under the entry deterrence strategy are

$$
\begin{aligned}
y_{L}^{d e t} & =\frac{\left(\beta_{1}+1\right) m_{X}}{\left(\beta_{1}-1\right) m_{I}}, \\
Q_{L}^{\text {det }} & =\frac{1}{\left(\beta_{1}+1\right) \eta} .
\end{aligned}
$$

The alternative for the leader is to apply an entry accommodation strategy, where it allows the follower to immediately invest once it has invested itself. Specifically, if the leader chooses its capacity $Q_{L} \leq \widehat{Q_{L}}(y)$, which will trigger the follower to make invest immediately. Theorem 5 gives the optimal investment decision of the leader when it uses the entry accommodation policy.

Theorem 5 The entry accommodation strategy will be considered if the current level of demand-to-cost ratio $y$ is larger than or equal to $y_{1}^{a c c}$, where

$$
y_{1}^{a c c}=\frac{\left[2\left(1-\beta_{1}\right)+\left(1+3 \beta_{1}\right) \kappa\right] m_{X}}{\left(\beta_{1}-1\right) m_{I}} .
$$

The value of the entry accommodation strategy, when investment takes place at $y$, is equal to

$$
g_{L}^{a c c}(y)=\frac{\left[y m_{I}-(2-\kappa) m_{X}\right]^{2}}{8 y \eta m_{X} m_{I}^{2}} .
$$

For the entry accommodation strategy, the optimal investment threshold and corresponding capacity level are given by

$$
\begin{aligned}
y_{L}^{a c c} & =\frac{\left(\beta_{1}+1\right)(2-\kappa) m_{X}}{\left(\beta_{1}-1\right) m_{I}}, \\
Q_{L}^{a c c} & \equiv Q_{L}^{a c c}\left(y_{L}^{a c c}\right)=\frac{1}{\left(\beta_{1}+1\right) \eta} .
\end{aligned}
$$

We find that the leader will make corresponding strategic adjustments when the current level $y$ is in different regions. For $y<y_{1}^{a c c}$, the leader will definitely apply an entry deterrence strategy. When $y \in\left(y_{1}^{a c c}, y_{2}^{\text {det }}\right)$, the leader chooses an entry deterrence or accommodation strategy that maximizes its value. Moreover, if $y>y_{2}^{d e t}$, the leader can only adopt the entry accommodation strategy. The leader will wait to invest until $y$ reaches $y_{L}^{\text {det }}$ for the first time. Accordingly, the leader's optimal investment strategy is summarized in the following Theorem.

Theorem 6 The optimal investment timing of the leader is

$$
y_{L}^{*}= \begin{cases}y_{L}^{\text {det }}, & y \in\left[0, y_{L}^{\text {det }}\right), \\ y, & y \in\left[y_{L}^{\text {det }}, \infty\right),\end{cases}
$$


and the corresponding optimal capacity level is

$$
Q_{L}^{*}(y)= \begin{cases}Q_{L}^{\text {det }}\left(y_{L}^{\text {det }}\right), & y \in\left[0, y_{L}^{\text {det }}\right), \\ Q_{L}^{\text {det }}(y), & y \in\left[y_{L}^{\text {det }}, \widehat{y}\right), \\ Q_{L}^{\text {acc }}(y), & y \in[\widehat{y}, \infty),\end{cases}
$$

where $\widehat{y}=\min \left\{y \in\left(y_{1}^{a c c}, y_{2}^{\text {det }}\right) \mid g_{L}^{a c c}(y)=g_{L}^{\text {det }}(y)\right\}$. Furthermore, the value of the leader is

$$
v_{L}^{*}(y)= \begin{cases}\left(\frac{y}{y_{L}^{\text {det }}}\right)^{\beta} g_{L}^{\text {det }}\left(y_{L}^{\text {det }}\right), & y \in\left[0, y_{L}^{\text {det }}\right), \\ g_{L}^{\text {det }}(y), & y \in\left[y_{L}^{\text {det }}, \widehat{y}\right), \\ g_{L}^{\text {acc }}(y), & y \in[\widehat{y}, \infty),\end{cases}
$$

\subsection{Symmetric costs}

In this subsection, we suppose that both firms have the same unit investment costs, that is, $I_{1 t}=I_{2 t}=I_{t}$. Considering the competition in the duopoly market, the firms might have incentives to preempt the market. Under the endogenous firm role, both firms have the opportunity to become market leader, and the advantage of becoming leader is that the firm can enjoy monopoly profit for a period of time. Thereafter, once it is known which of the two firms invests first, the other will become a follower. After the first investor invests, the second investor behaves as if the market position is exogenous, because its investment decision no longer involve strategic aspects. Therefore, we can refer to Theorem 3 (with $\kappa=1$ ) for the second investor's optimal investment decision in the case of endogenous firm roles.

The preemption threshold, denoted by $y_{L e}^{*}$, is the moment for which the firms are indifference between being a leader or a follower. Fudenberg and Tirole [27] first studied the preemptive trigger decision of two firms within a deterministic framework. Following [30], we can obtain the preemptive threshold in stochastic timing game by solving the following equation for $y_{L e}^{*}$

$$
v_{F}^{*}\left(y_{L e}^{*}, Q_{L}^{*}\left(y_{L e}^{*}\right)\right)=v_{L}^{*}\left(y_{L e}^{*}\right) .
$$

The intuition behind (52) is that when $y<y_{L e}^{*}$, no firm wants to invest since in this case it would be more profitable to be a follower (because the leader's value is lower than the follower's value). On the other hand, when $y>y_{L e}^{*}$, the return of first investor is bigger, so it is more profitable for a firm to invest immediately and become a leader than to wait for investment. Suppose that firm 1 wants to invest at level $y$, then firm 2 would preempt firm 1 and invest at $y-\epsilon$, which will cause firm 1 to invest even earlier, at $y-2 \epsilon$. This preemptive mechanism continues until $y-n \epsilon=y_{L e}^{*}$, where one of the firms will invest. Because the firms are symmetric, they have equal chances of becoming market leader at the preemptive trigger. The result of the preemption game: the first investor, as the leader, invests at the preemptive equilibrium point $y_{L e}^{*}$ in capacity $Q_{L e}^{*}=Q_{L}^{*}\left(y_{L e}^{*}\right)$, and the second investor, as the follower, invests at $y_{F e}^{*}=y_{F}^{*}\left(Q_{L}^{*}\left(y_{L e}^{*}\right)\right)$ in capacity $Q_{F e}^{*}=Q_{F}^{*}\left(y_{L e}^{*}\right)$.

\subsection{Welfare analysis}

Now we proceed to the welfare analysis in a duopoly market. In order to examine the investment outcome of the duopoly from the perspective of social welfare, we suppose a social 
planner who is allowed to make an investment at two moments in time. For these two investments, the social planner is free to choose the investment timing and capacity size. We use backward induction to solve the social optimal investment strategy.

In order to compare the investment behavior of the monopolistic firm and the social planner, we first briefly examine the impact on investment policy when the monopolistic firm has two investment opportunities. For the monopolist problem, the first investment increases the firm's capacity from 0 to $Q_{1}$, and the second one from $Q_{1}$ to $Q_{1}+Q_{2}$. We solve the problem backwards. Firstly, for a given capacity level $Q_{1}$, the second investment is solved. Thereafter, given the optimal investment behavior of the second investment, we solve the first investment. When a monopolistic firm can invest twice in time, the following theorem describes its optimal investment strategy.

Theorem 7 In a market with multiplicative demand, if a monopolist has two investment opportunities, then the optimal investment thresholds $y_{1}^{*}, y_{2}^{*}$ and the corresponding optimal capacities $Q_{1}^{*}, Q_{2}^{*}$ are implicitly determined by the following equations:

$$
\begin{aligned}
& 1-\frac{\beta_{1} \eta Q_{1}^{*}}{1-\eta Q_{1}^{*}}-2\left[\frac{\beta_{1}\left(1-2 \eta Q_{1}^{*}\right)}{\left(\beta_{1}+1\right)\left(1-\eta Q_{1}^{*}\right)}\right]^{\beta_{1}}=0, \\
& y_{1}^{*}\left(Q_{1}\right)=\frac{\beta_{1} m_{X}}{\left(\beta_{1}-1\right)\left(1-\eta Q_{1}\right) m_{I}} . \\
& y_{2}^{*}\left(Q_{1}\right)=\frac{\left(\beta_{1}+1\right) m_{X}}{\left(\beta_{1}-1\right)\left(1-2 \eta Q_{1}\right) m_{I}}, \\
& Q_{2}^{*}\left(Q_{1}\right)=\frac{1-2 \eta Q_{1}}{\left(\beta_{1}+1\right) \eta} .
\end{aligned}
$$

Now we consider the optimization problem of the social planner. Regarding the second investment, we know that the follower's investment decision in the duopoly model is essentially the same as the monopolist's investment decision. Therefore, similar to the monopoly model in Sect. 3, if we assume that we have the same quantity of the first investment in the case of social welfare and duopoly, then the capacity level of the second investment in the welfare maximization policy will be twice as much as the capacity chosen by the follower. Let $y_{F, W}^{*}$ denote the investment threshold of the second investment in the welfare maximizing policy, and the corresponding optimal capacity level is denoted by $Q_{F, W}^{*}$. Following [11], we can derive that

$$
\begin{array}{r}
y_{F, W}^{*}\left(Q_{L}\right)=\frac{\left(\beta_{1}+1\right) m_{X}}{\left(\beta_{1}-1\right)\left(1-\eta Q_{L}\right) m_{I}}, \\
Q_{F, W}^{*}\left(Q_{L}\right)=\frac{2\left(1-\eta Q_{L}\right)}{\left(\beta_{1}+1\right) \eta} .
\end{array}
$$

As for the first investment, according to Theorem 7, the capacity level for maximizing welfare, which is denoted by $Q_{L, W}^{*}$, is twice the capacity chosen by the monopolist who can make two investments. This capacity level is the solution of the following equation:

$$
1-\frac{1 / 2 \beta_{1} \eta Q_{L, W}^{*}}{1-1 / 2 \eta Q_{L, W}^{*}}-2\left[\frac{\beta_{1}\left(1-\eta Q_{L, W}^{*}\right)}{\left(\beta_{1}+1\right)\left(1-1 / 2 \eta Q_{L, W}^{*}\right)}\right]^{\beta_{1}}=0 .
$$

The optimal trigger for the first investment of the social planner is given by:

$$
y_{L, W}^{*}\left(Q_{L}\right)=\frac{\beta_{1} m_{X}}{\left(\beta_{1}-1\right)\left(1-1 / 2 \eta Q_{L}\right) m_{I}} .
$$


Table 1 Related variables and numerical assumptions

Basic parameters: $r=0.06, \eta=0.05, \mu_{X}=0.038, \mu_{I}=0.028, \sigma_{X}=0.3, \sigma_{I}=0.2, \lambda=0.1, \sigma_{1}=0.1$, $\sigma_{2}=0.1, S k_{1}=0, S k_{2}=0$.

Huisman \& Kort (2015): demand uncertainty model without jump ( $\left.\mu_{I}=\sigma_{I}=\lambda=0\right)$. two-factor diffusion model: demand and investment cost uncertainties model without jumps $(\lambda=0)$. $\mu_{1}=0.1, \mu_{2}=-0.1$ : upward jump in demand and downward jump in investment cost. $\mu_{1}=-0.1, \mu_{2}=0.1$ : downward jump in demand and upward jump in investment cost.

Consequently, if the capacity level in the case of welfare maximizing is twice that of the monopolist who has two opportunities to invest, then the investment thresholds of welfare maximizing policy are the same as that of the monopolist. According to Eqs. (57) and (58), it can be found that the second welfare investment will occur later than the follower's investment in the duopoly model, and the corresponding capacity size will be less than twice as high. Furthermore, from a welfare point of view, the firms invest too early in too small capacities in the duopoly of symmetric costs.

The total social welfare is given by

$$
\begin{gathered}
S W\left(y_{L}, Q_{L}, y_{F}, Q_{F}, y\right)=\left(\frac{y}{y_{L}}\right)^{\beta_{1}}\left(\frac{y_{L} Q_{L}\left(2-\eta Q_{L}\right)}{2 m_{X}}-\frac{Q_{L}}{m_{I}}\right)+\left(\frac{y}{y_{F}}\right)^{\beta_{1}} \\
\left(\frac{y_{F}\left(Q_{L}+Q_{F}\right)\left(2-\eta\left(Q_{L}+Q_{F}\right)\right)}{2 m_{X}}-\frac{Q_{F}}{m_{I}}-\frac{y_{F} Q_{L}\left(2-\eta Q_{L}\right)}{2 m_{X}}\right) .
\end{gathered}
$$

which obviously depends on the investment moments, $y_{L}$ and $y_{F}$, the capacities $Q_{L}$ and $Q_{F}$ chosen by the leader and the follower, and the level of demand-to-cost ratio $y$.

\section{Numerical illustration}

In this section, we perform a numerical analysis to investigate the impact of combining uncertainty about market demand and investment cost on investment decision of firms. For the numerical illustration, we assume that the discontinuous jumps in demand shock and unit investment cost in our model are caused by the same sudden events, so they have the same jump intensity, i.e., $\lambda=\lambda_{X}=\lambda_{I}$.

The basic parameter values of numerical simulation are given in Table 1 . When analyzing the influence of the change of a specific jump parameter on the optimal investment decision, we assum that other parameters remain unchanged.

\subsection{Monopoly case}

We first examine the impact of $\sigma_{X}$ and $\sigma_{I}$ on the optimal investment threshold $y^{*}$ and capacity $Q^{*}$ for four different cases in the monopoly market. As shown in Fig. 1, we observe that $y^{*}$ and $Q^{*}$ both increases with $\sigma_{X}$ and $\sigma_{I}$. This implies that a higher uncertainty of market demand or unit investment cost delays the investment but promotes a larger scale production capacity. In other words, a monopolistic firm will adopt a waiting strategy and choose a larger capacity level to make greater profits in a more volatile market. This is consistent with the conclusion in [11]. It is worth noting that in the Huisman \& Kort (2015) model ( [11]), the unit investment cost is a constant $\left(\sigma_{I}=0\right)$, so the optimal investment timing and optimal 

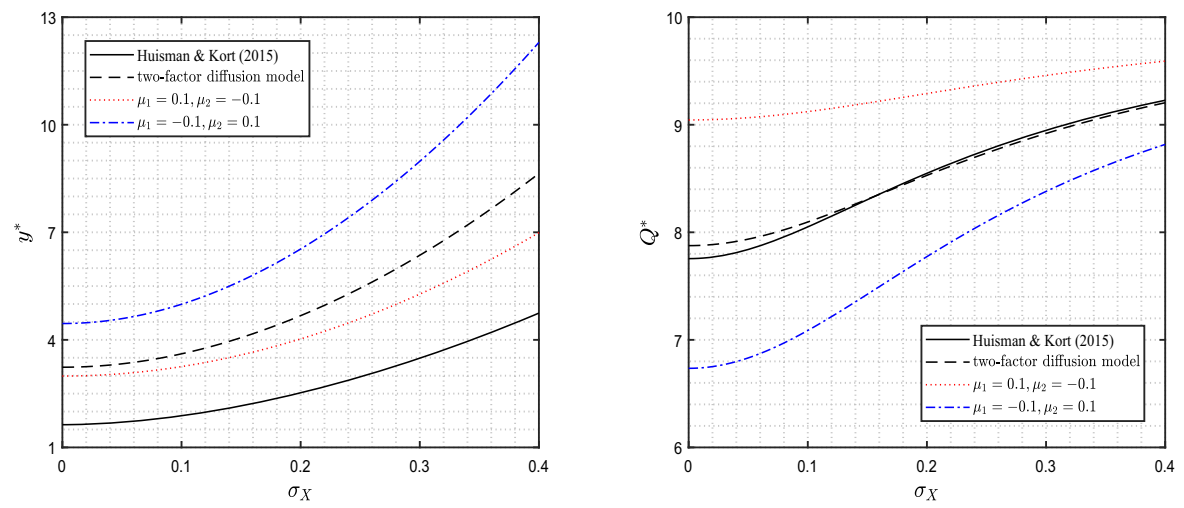

(a) Optimal investment threshold and capacity as function of $\sigma_{X}$.
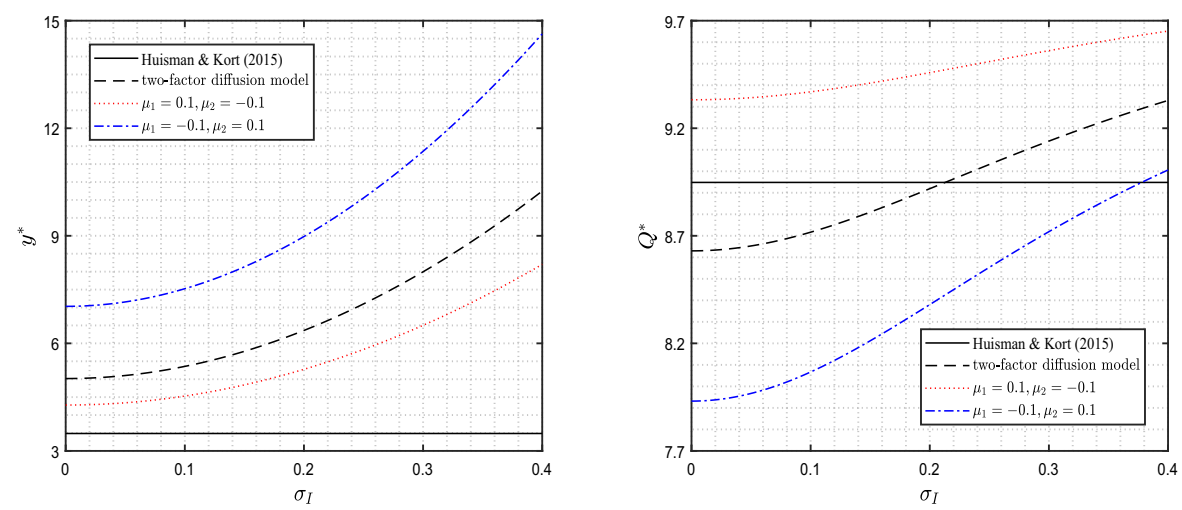

(b) Optimal investment threshold and capacity as function of $\sigma_{I}$.

Fig. 1 Optimal investment decisions of monopolist for different uncertainties of market demand and investment cost

capacity depend only on market demand and are not affected by the change of investment cost.

The top-left and bottom-left panels in Fig. 1 show that when the market demand and investment cost of the project are both diffusion processes (jump is not considered), the optimal investment threshold of the monopolistic firm is larger than that of Huisman \& Kort (2015) model. Moreover, if we assume that both $\boldsymbol{X}$ and $\boldsymbol{I}$ evolve according to the jumpdiffusion processes, the optimal investment threshold of the monopolistic firm is also larger than that of Huisman \& Kort (2015) model. However, the top-right and bottom-right panels in Fig. 1 illustrate the relationship of optimal investment capacities between the Huisman \& Kort and two-factor diffusion models is ambiguous. According to Eq. (21), the monopolist's optimal investment capacity $Q^{*}$ depends on the value of $\beta_{1}$, and the larger $\beta_{1}$ is, the smaller $Q^{*}$ is. However, the value of $\beta_{1}$ in Huisman \& Kort (2015) model is given by

$$
\frac{1}{2}-\frac{\mu_{X}}{\sigma_{X}^{2}}+\sqrt{\left(\frac{1}{2}-\frac{\mu_{X}}{\sigma_{X}^{2}}\right)^{2}+\frac{2 r}{\sigma_{X}^{2}}},
$$


whileas, from Eq. (17), the value of $\beta_{1}$ in the two-factor diffusion model is equal to

$$
\frac{1}{2}-\frac{\mu_{X}-\mu_{I}}{\sigma_{X}^{2}+\sigma_{I}^{2}}+\sqrt{\left(\frac{1}{2}-\frac{\mu_{X}-\mu_{I}}{\sigma_{X}^{2}+\sigma_{I}^{2}}\right)^{2}+\frac{2\left(r-\mu_{I}\right)}{\sigma_{X}^{2}+\sigma_{I}^{2}}}
$$

Obviously, the $\beta_{1}$ of these two models cannot be compared. Therefore, the relationship between the optimal investment capacities of the two models is ambiguous.

In addition, from Fig. 1, we can derive that, compared to the two-factor diffusion processes, in the two-factor jump-diffusion processes, the upward jump in demand and downward jump in investment cost enable the monopoly firm to accelerate investment with greater capacity. On the other hand, the downward jump in demand and upward jump in investment cost enable the monopoly firm to delay investment with smaller capacity. This is due to the fact that when demand jumps up and investment cost jumps down, the NPV of the investment project will increase, whereas when demand jumps up and investment cost jumps down, the NPV of the investment project will decrease.

\subsection{Duopoly case}

We now proceed to investigate the optimal investment decisions in a duopoly market. Table 2 lists the optimal investment thresholds and optimal capacities of tow firms with different levels of $\sigma_{X}$ and $\sigma_{I}$. Specifically, Table 2(a) reports the optimal investment strategies in an asymmetric duopoly market $(\kappa=1.5)$, while Table $2(\mathrm{~b})$ reports preemption equilibrium in a symmetric duopoly market $(\kappa=1)$ by solving Eq. (52). We see from Table 2 that the optimal investment thresholds and optimal capacities of both the leader and the follower increase with each parameter in $\sigma_{X}$ and $\sigma_{I}$. This confirms Huisman \& Kort ([11]), who concludes that when the economic environment becomes more uncertain, both firms will delay investment with greater capacity.

Furthermore, as seen in Table 2, in contrast to the Huisman \& Kort's model, in our twofactor diffusion model, both the leader and the follower will delay investment as the $\sigma_{X}$ or $\sigma_{I}$ increases, but the relationship between the optimal investment capacities of these two models is ambiguous.

As we expected, Table 2 states that, compared to the two-factor diffusion processes, in the two-factor jump-diffusion processes, if the demand jumps up and investment cost jumps down, both the leader and the follower will invest earlier in more capacity, whileas if the jumps in demand are downwards and the jumps in investment cost are upwards, the duopolistic firms will invest later in less capacity. This is because the upward jump in demand and downward jump in investment cost indicate that the economic environment is upbeat, which encourages the leader and the follower invest earlier with higher capacities, and vice versa. Finally, from Table 2, we conclude that the first investor should invest earlier in a higher capacity than the second investor due to the threat of pre-emption.

\section{Concluding remarks}

In this paper, we investigate the impact of unexpected sudden events on the optimal investment thresholds and optimal capacities in a duopoly market by using the real option game method. Based on the duopoly game model, we introduce the Poisson jump process to describe the effect of sudden events on the product market. Since our jump-diffusion model allows us to 


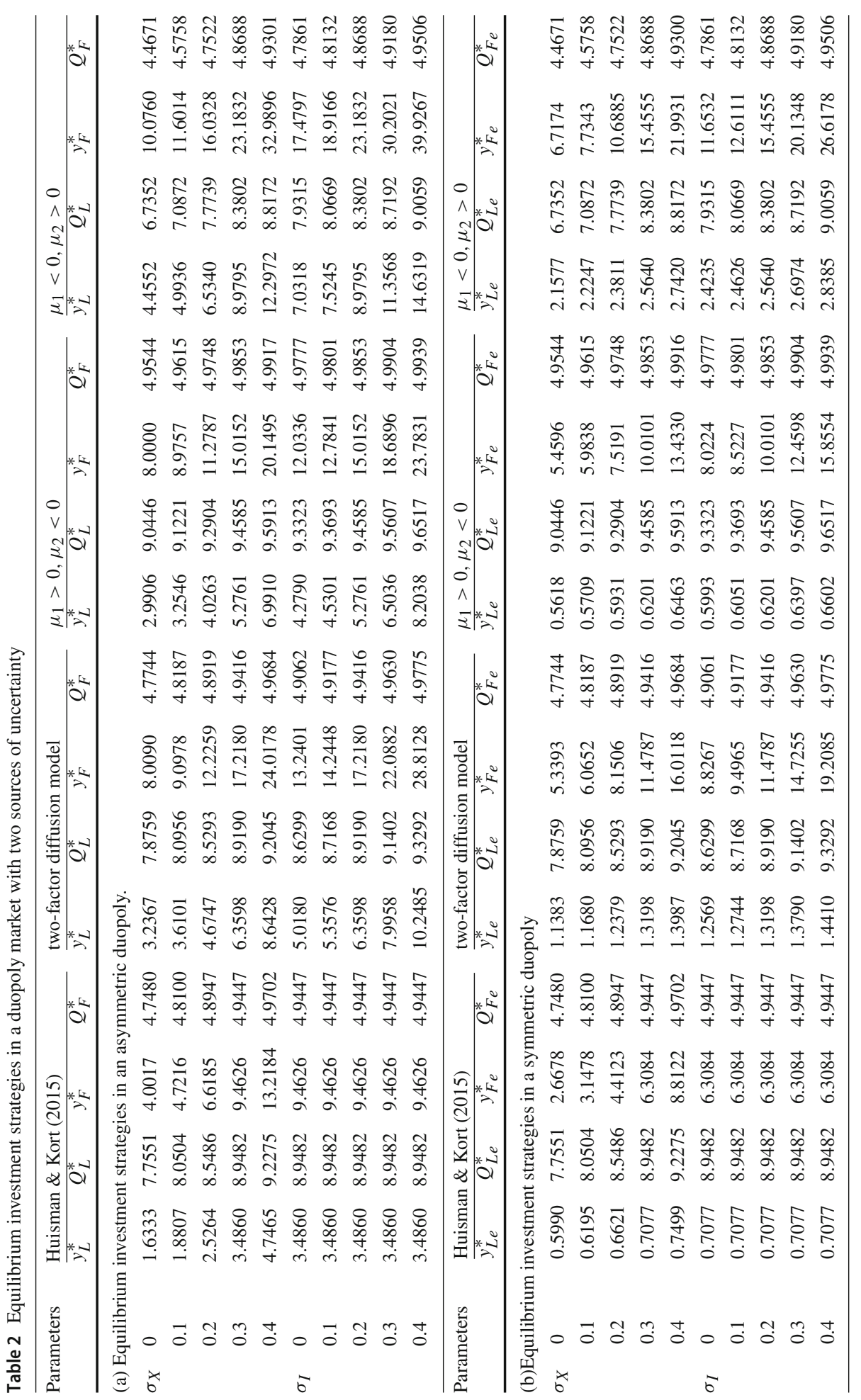


investigate the duopoly competition in a more realistic economic setting, therefore, we are likely to obtain more accurate and more reliable results than those reported by [11].

We find that whether in the monopoly or duopoly market, uncertainty of market has a positive impact on the optimal investment timing and optimal capacity. Based on the twofactor jump-diffusion model, this paper demonstrates that the sudden events will affect the investment thresholds and production capacities of the firms. More specifically, compared to the two-factor diffusion processes, if the jumps in demand are upwards and the jumps in investment cost are downwards, the duopolistic firms will invest earlier in higher capacity, and vice versa. Additionally, in order to capture the market, the optimal investment threshold for the first investor in a symmetric duopoly is relatively smaller than that of the first investor in an asymmetric duopoly. Therefore, when the market changes abruptly due to sudden events, the firm can apply the jump-diffusion model to analyze the project's optimal investment decisions and adjust its strategies in time to avoid unnecessary losses or miss the investment opportunities.

Our model studies the investment decisions in a duopoly market with two-factor uncertainties, both following jump-diffusion processes. However, investment in practice is much more complex. Several extensions are possible for this paper. For instance, the competition usually involves more than two firms and a third player can adopt either a follower, leader or mixed strategy (see [31]). A direction for future research is to study the optimal investment strategy of the middle player. Moreover, in order to understand whether the results are sensitive to the choice of specific demand structure, or if they can be extended, it is also interesting to investigate different demand models. Finally, the firms only invest once in our model. If the firms can invest multiple times, that is, have several investment options, future research should address their strategic interactions.

Funding This research was funded by the National Natural Science Foundation of China (No. 71361003) and Natural Science Foundation of Guizhou Province (No. [2018]3002).

\section{Declarations}

Conflict of interest The authors declare that there are no conflicts of interest regarding the publication of this paper.

Availability of data and material All data generated or analyzed during this study are included in this paper.

\section{Appendix}

\section{Proof of Theorem 1}

Using a change of variable in the performance function, we obtain

$$
J(x, i, Q)=\mathbb{E}_{(x, i)}\left[e^{-r \tau}\left(\int_{0}^{\infty} e^{-r t}\left(X_{t+\tau} Q(1-\eta Q)-I_{t+\tau} Q\right) d t\right)\right] .
$$

According to the tower property of conditional expectation and strong Markov property, we have

$$
J(x, i, Q)=\mathbb{E}_{(x, i)}\left\{e^{-r \tau} \mathbb{E}\left[\int_{0}^{\infty} e^{-r t}\left(X_{t+\tau} Q(1-\eta Q)-I_{t+\tau} Q\right) d t \mid\left(X_{\tau}, I_{\tau}\right)\right]\right\}
$$


where

$$
\begin{aligned}
& \mathbb{E}\left[\int_{0}^{\infty} e^{-r t}\left(X_{t+\tau} Q(1-\eta Q)-I_{t+\tau} Q\right) d t \mid\left(X_{\tau}, I_{\tau}\right)\right] \\
= & \int_{0}^{\infty} e^{-r t}\left(Q(1-\eta Q) \mathbb{E}\left(X_{t+\tau} \mid X_{\tau}\right)-Q \mathbb{E}\left(I_{t+\tau} \mid I_{\tau}\right)\right) d t \\
= & \frac{X_{\tau} Q(1-\eta Q)}{r-\mu_{X}-\lambda_{X} \mu_{1}}-\frac{I_{\tau} Q}{r-\mu_{I}-\lambda_{I} \mu_{2}} .
\end{aligned}
$$

Substituting (A3) into (A2) yields Eq. (7).

\section{Proof of Theorem 2}

Given the demand shock and the unit investment cost are $x$ and $i$, respectively, if the firm's investment capacity is $Q$, then the profit of the firm is equal to,

$$
G(x, i, Q)=\frac{x Q(1-\eta Q)}{m_{X}}-\frac{i Q}{m_{I}} .
$$

Let $g(y, Q)=G(x, i, Q) / i$, the firm's profit can be rewritten as

$$
g(x, i, Q)=\frac{y Q(1-\eta Q)}{m_{X}}-\frac{Q}{m_{I}} .
$$

Maximize (A5) with respect to $Q$ yields the optimal capacity level $Q^{*}$

$$
Q^{*}(y)=\frac{1}{2 \eta}\left(1-\frac{m_{X}}{y m_{I}}\right) .
$$

Furthermore, the value of the firm, $v(y)$, is given by the partial differential equation (15), with the boundary conditions

$$
\begin{aligned}
& A_{1} y^{* \beta_{1}}=\frac{y^{*} Q(1-\eta Q)}{m_{X}}-\frac{Q}{m_{I}}, \\
& \beta_{1} A_{1} y^{* \beta_{1}-1}=\frac{Q(1-\eta Q)}{m_{X}},
\end{aligned}
$$

where $\beta_{1}$ is the positive solution of the nonlinear equation (17).

The value of $\beta_{1}$ can be obtained by numerically solving Eq. (17). Combining (A7) and (A8) yields Eq. (20). From (A6) and Eq. (20), we can obtain the results in Theorem 2.

\section{Proof of Theorem 3}

Given the current level of the stochastic demand and unit investment cost denoted by $x$ and $i$, and the capacity level $Q_{L}$ and $Q_{F}$ of the leader and the follower, respectively, the profit of the follower is given by

$$
G_{F}\left(x, i, Q_{L}, Q_{F}\right)=\frac{x Q_{F}\left(1-\eta\left(Q_{L}+Q_{F}\right)\right)}{m_{X}}-\frac{\kappa i Q_{F}}{m_{I}} .
$$


Further, let $G_{F}\left(x, i, Q_{L}, Q_{F}\right)=i g_{F}\left(y, Q_{L}, Q_{F}\right)$, then the follower's profit can be rewritten as

$$
g_{F}\left(y, Q_{L}, Q_{F}\right)=\frac{y Q_{F}\left(1-\eta\left(Q_{L}+Q_{F}\right)\right)}{m_{X}}-\frac{\kappa Q_{F}}{m_{I}} .
$$

Maximizing (A10) with respect to $Q_{F}$ yields the optimal capacity of the follower

$$
Q_{F}^{*}\left(y, Q_{L}\right)=\frac{1}{2 \eta}\left(1-\eta Q_{L}-\frac{\kappa m_{X}}{y m_{I}}\right) .
$$

Before the follower has invested, i.e., $y<y_{F}^{*}\left(Q_{L}\right)$, the value function of the follower is

$$
v_{F}(X)=A_{F} y^{\beta_{1}},
$$

and it satisfies the following value matching and smooth pasting conditions

$$
\begin{aligned}
& v_{F}\left(y_{F}^{*}\right)=\frac{y_{F}^{*} Q_{F}\left(1-\eta\left(Q_{L}+Q_{F}\right)\right)}{m_{X}}-\frac{\kappa Q_{F}}{m_{I}}, \\
& v_{F}^{\prime}\left(y_{F}^{*}\right)=\frac{Q_{F}\left(1-\eta\left(Q_{L}+Q_{F}\right)\right)}{m_{X}} .
\end{aligned}
$$

Solving the above two equations, we obtain

$$
\begin{array}{r}
y_{F}^{*}\left(Q_{L}, Q_{F}\right)=\frac{\beta_{1}}{\beta_{1}-1} \frac{\kappa m_{X}}{\left(1-\eta\left(Q_{L}+Q_{F}\right)\right) m_{I}}, \\
A_{F}\left(Q_{L}\right)=\frac{1-\eta Q_{L}}{\left(\beta_{1}^{2}-1\right) m_{I} \eta}\left[\frac{\left(\beta_{1}-1\right)\left(1-\eta Q_{L}\right) m_{I}}{\left(\beta_{1}+1\right) \kappa m_{X}}\right]^{\beta_{1}} .
\end{array}
$$

According to (A11) and (A15), the optimal threshold and capacity level of the follower are Eqs. (35) and (36) respectively.

\section{Proof of Theorem 4}

For the entry deterrence strategy, the leader's value function at the moment of investment can be expressed as

$$
g_{L}^{\text {det }}\left(y, Q_{L}\right)=\frac{y\left(1-\eta Q_{L}\right) Q_{L}}{m_{X}}-\frac{Q_{L}}{m_{I}}-\left(\frac{y}{y_{F}^{*}\left(Q_{L}\right)}\right)^{\beta_{1}} \frac{y_{F}^{*}\left(Q_{L}\right) \eta Q_{F}^{*}\left(Q_{L}\right) Q_{L}}{m_{X}} .
$$

Substituting (35) and (36) into (A17) yields the following equation

$$
g_{L}^{d e t}\left(y, Q_{L}\right)=\frac{y Q_{L}\left(1-\eta Q_{L}\right)}{m_{X}}-\frac{Q_{L}}{m_{I}}-\left(\frac{y\left(\beta_{1}-1\right)\left(1-\eta Q_{L}\right) m_{I}}{\left(\beta_{1}+1\right) \kappa m_{X}}\right)^{\beta_{1}} \frac{\kappa Q_{L}}{\left(\beta_{1}-1\right) m_{I}} .
$$

Maximizing $g_{L}^{\text {det }}\left(y, Q_{L}\right)$ with respect to $Q_{L}$ gives the following first-order necessary condition

$$
\begin{aligned}
\phi\left(y, Q_{L}\right) \equiv & \frac{y\left(1-2 \eta Q_{L}\right)}{m_{X}}-\frac{1}{m_{I}}-\left[\frac{y\left(\beta_{1}-1\right)\left(1-\eta Q_{L}\right) m_{I}}{\left(\beta_{1}+1\right) \kappa m_{X}}\right]^{\beta_{1}} \\
& \times \frac{\kappa\left(1-\left(\beta_{1}+1\right) \eta Q_{L}\right)}{\left(\beta_{1}-1\right)\left(1-\eta Q_{L}\right) m_{I}}=0 .
\end{aligned}
$$


Solving (A19) yields $Q_{L}^{\text {det }}(y)$. Let $Q_{L}=0$ in (A19) gives Eq. (39). Further, let

$$
\psi(y) \equiv \phi\left(y, Q_{L}=0\right)=\frac{y}{m_{X}}-\frac{1}{m_{I}}-\left[\frac{y\left(\beta_{1}-1\right) m_{I}}{\left(\beta_{1}+1\right) \kappa m_{X}}\right]^{\beta_{1}} \frac{\kappa}{\left(\beta_{1}-1\right) m_{I}},
$$

then we get

$$
\begin{aligned}
& \psi(0)=-\frac{1}{m_{I}}<0 \\
& \psi\left(y_{F}^{*}(0)\right)=\frac{1}{m_{I}}\left(\frac{\beta_{1} \kappa}{\beta_{1}-1}-1\right)=\frac{\beta_{1}(\kappa-1)+1}{\left(\beta_{1}-1\right) m_{I}}>0, \\
& \frac{\partial \psi(y)}{\partial y}=\frac{1}{m_{X}}\left[1-\frac{\beta_{1}}{\beta_{1}+1}\left(\frac{y}{y_{F}^{*}(0)}\right)^{\beta_{1}-1}\right] .
\end{aligned}
$$

For $y \in\left(0, y_{F}^{*}(0)\right)$, then $\partial \psi(y) / \partial y>0$ holds. Therefore $y_{1}^{\text {det }}$ exists. In addition, the leader can no longer uses the entry deterrence strategy if $y \geq y_{F}^{*}\left(Q_{L}^{\text {det }}(y)\right)$.

We define $y_{2}^{\text {det }}$ as

$$
y_{2}^{\text {det }}=y_{F}^{*}\left(Q_{L}^{\text {det }}\left(y_{2}^{\text {det }}\right)\right) .
$$

Substitution of (35) into (A19) results in

$$
\frac{\beta_{1}+1}{\beta_{1}-1} \frac{1-2 \eta Q_{L}}{1-\eta Q_{L}} \kappa-1-\frac{\left(1-\left(\beta_{1}+1\right) \eta Q_{L}\right)}{\left(\beta_{1}-1\right)\left(1-\eta Q_{L}\right)} \kappa=0 .
$$

Solving Eq. (A25) for $Q_{L}$, we obtain

$$
Q_{L}=\frac{\beta_{1} \kappa-\left(\beta_{1}-1\right)}{\left[\beta_{1}(\kappa-1)+\kappa+1\right] \eta} .
$$

By substituting (A26) into (35), we can obtain Eq. (40).

The option value of the leader is given by

$$
v_{L}^{d e t}(y)=A_{L}^{d e t} y^{\beta_{1}}
$$

To determine $y_{L}^{\text {det }}$, the corresponding value matching and smooth pasting conditions are given by

$$
\begin{aligned}
& A_{L}^{d e t} y^{\beta_{1}}=\frac{y\left(1-\eta Q_{L}(y)\right) Q_{L}(y)}{m_{X}}-\frac{Q_{L}(y)}{m_{I}}-\left[\frac{y\left(\beta_{1}-1\right)\left(1-\eta Q_{L}(y)\right) m_{I}}{\left(\beta_{1}+1\right) \kappa m_{X}}\right]^{\beta_{1}} \frac{\kappa Q_{L}(y)}{\left(\beta_{1}-1\right) m_{I}}, \\
& \beta_{1} A_{L}^{d e t} y^{\beta_{1}-1}=\frac{Q_{L}(y)\left(1-\eta Q_{L}(y)\right)+y \frac{\partial Q_{L}}{\partial y}\left(1-2 \eta Q_{L}(y)\right)}{m_{X}}-\frac{1}{m_{I}} \frac{\partial Q_{L}}{\partial y}- \\
& \left(\frac{y\left(\beta_{1}-1\right)\left(1-\eta Q_{L}(y)\right) m_{I}}{\left(\beta_{1}+1\right) \kappa m_{X}}\right)^{\beta_{1}} \frac{\left[Q_{L}(y)\left(\beta_{1}\left(1-\eta Q_{L}(y)\right)-\left(\beta_{1}+1\right) y \eta \frac{\partial Q_{L}}{\partial y}\right)+y \frac{\partial Q_{L}}{\partial y}\right] \kappa}{y\left(\beta_{1}-1\right)\left(1-\eta Q_{L}(y)\right) m_{I}} .
\end{aligned}
$$


Substitution of (A29) into (A28) results in

$$
\begin{aligned}
& \frac{y Q_{L}(y)\left(1-\eta Q_{L}(y)\right)}{m_{X}}-\frac{y Q_{L}(y)\left(1-\eta Q_{L}(y)\right)+y^{2} \frac{\partial Q_{L}}{\partial y}\left(1-2 \eta Q_{L}(y)\right)}{\beta_{1} m_{X}}-\frac{Q_{L}(y)}{m_{I}}+ \\
& \frac{y}{\beta_{1} m_{I}} \frac{\partial Q_{L}}{\partial y}+\left(\frac{y\left(\beta_{1}-1\right)\left(1-\eta Q_{L}(y)\right) m_{I}}{\left(\beta_{1}+1\right) \kappa m_{X}}\right)^{\beta_{1}}\left(\frac{y \frac{\partial Q_{L}}{\partial y}\left(1-\left(\beta_{1}+1\right) \eta Q_{L}(y)\right) \kappa}{\beta_{1}\left(\beta_{1}-1\right)\left(1-\eta Q_{L}(y)\right) m_{I}}\right)=0 .
\end{aligned}
$$

Combining (A19) and (A30) leads to

$$
\frac{\left(\beta_{1}-1\right) y\left(1-\eta Q_{L}\right)}{m_{X}}-\frac{\beta_{1}}{m_{I}}=0 .
$$

Solving Eq. (A31), the leader's threshold $y_{L}^{\text {det }}$ is equal to

$$
y_{L}^{\text {det }}\left(Q_{L}\right)=\frac{\beta_{1} m_{X}}{\left(\beta_{1}-1\right)\left(1-\eta Q_{L}\right) m_{I}} .
$$

Substituting (A32) into (A19), the optimal investment threshold and optimal capacity of the leader are, respectively, given by Eq. (43) and Eq. (44) in Theorem 4.

\section{Proof of Theorem 5}

For the entry accommodation strategy, the value function of the leader at the moment of investment is given by

$$
g_{L}^{a c c}\left(y, Q_{L}\right)=\frac{y Q_{L}\left(1-\eta\left(Q_{L}+Q_{F}^{*}\left(Q_{L}\right)\right)\right)}{m_{X}}-\frac{Q_{L}}{m_{I}} .
$$

Substituting (A11) into (A33) and maximize with respect to $Q_{L}$ gives

$$
Q_{L}^{a c c}(y)=\frac{1}{2 \eta}\left(1-\frac{(2-\kappa) m_{X}}{y m_{I}}\right) .
$$

The leader will adopt the accommodation strategy only if the optimal quantity $\left.Q_{L}^{a c c}(y)\right)$ causes the follower to invest immediately. Thus it holds that

$$
y \geq y_{F}^{*}\left(Q_{L}^{a c c}(y)\right),
$$

We define $y_{1}^{a c c}$ as

$$
y_{1}^{a c c}=y_{F}^{*}\left(Q_{L}^{a c c}\left(y_{1}^{a c c}\right)\right) .
$$

Then, substitution of (36) and (A34) into (A36) yields Eq. (45).

Furthermore, by substituting (36) and (A34) into (A33), it gives Eq.(46). The value matching and smooth pasting conditions for the entry accommodation strategy are given by

$$
\begin{aligned}
& A_{L}^{a c c} y^{\beta_{1}}=\frac{\left[y m_{I}-(2-\kappa) m_{X}\right]^{2}}{8 y \eta m_{X} m_{I}^{2}}, \\
& \beta_{1} A_{L}^{a c c} y^{\beta_{1}-1}=\frac{y^{2} m_{I}^{2}-(2-\kappa)^{2} m_{X}^{2}}{8 y^{2} \eta m_{X} m_{I}^{2}} .
\end{aligned}
$$


Substituting (A38) into (A37) leads to

$$
\frac{\left[y m_{I}-(2-\kappa) m_{X}\right]^{2}}{8 y \eta m_{X} m_{I}^{2}}-\frac{y^{2} m_{I}^{2}-(2-\kappa)^{2} m_{X}^{2}}{8 \beta_{1} y \eta m_{X} m_{I}^{2}}=0,
$$

and after some simple calculations, Equation (A39) can be reformulated as

$$
\frac{\left[y m_{I}-(2-\kappa) m_{X}\right]\left[\left(\beta_{1}-1\right) y m_{I}-\left(\beta_{1}+1\right)(2-\kappa) m_{X}\right]}{8 \beta_{1} y \eta m_{X} m_{I}^{2}}=0 .
$$

Acoording to Eq. (A37), $y=\left[(2-\kappa) m_{X}\right] / m_{I}$ is not a valid solution to Eq. (A40). Hence, we obtain

$$
y_{L}^{a c c}=\frac{\left(\beta_{1}+1\right)(2-\kappa) m_{X}}{\left(\beta_{1}-1\right) m_{I}},
$$

and substitution of (A41) into (A34) gives Eq. (48) in Theorem 5.

\section{Proof of Theorem 6}

Given in the text.

\section{Proof of Theorem 7}

When the firm's capacity increases from $Q_{1}$ to $Q_{1}+Q_{2}$, the value of the firm at the second investment is equal to

$$
G_{2}\left(x, i, Q_{1}, Q_{2}\right)=\frac{x\left(Q_{1}+Q_{2}\right)\left(1-\eta\left(Q_{1}+Q_{2}\right)\right)}{m_{X}}-\frac{i Q_{2}}{m_{I}} .
$$

Let $G_{2}\left(x, i, Q_{L}, Q_{F}\right)=i g_{2}\left(y, Q_{L}, Q_{F}\right)$, the value of the firm at the moment of the second investment can be rewritten as

$$
g_{2}\left(y, Q_{1}, Q_{2}\right)=\frac{y\left(Q_{1}+Q_{2}\right)\left(1-\eta\left(Q_{1}+Q_{2}\right)\right)}{m_{X}}-\frac{Q_{2}}{m_{I}} .
$$

Before the second investment, the value of the firm is

$$
v_{2}\left(y, Q_{1}\right)=\frac{y Q_{1}\left(1-\eta Q_{1}\right)}{m_{X}}+B_{2} y^{\beta_{1}},
$$

In addition, the value-matching and smooth-pasting conditions are as follows

$$
\begin{aligned}
& \frac{y_{2}^{*} Q_{1}\left(1-\eta Q_{1}\right)}{m_{X}}+B_{2} y_{2}^{* \beta_{1}}=\frac{y_{2}^{*}\left(Q_{1}+Q_{2}\right)\left(1-\eta\left(Q_{1}+Q_{2}\right)\right)}{m_{X}}-\frac{Q_{2}}{m_{I}} . \\
& \frac{Q_{1}\left(1-\eta Q_{1}\right)}{m_{X}}+\beta_{1} B_{2} y_{2}^{* \beta_{1}-1}=\frac{\left(Q_{1}+Q_{2}\right)\left(1-\eta\left(Q_{1}+Q_{2}\right)\right)}{m_{X}} .
\end{aligned}
$$

Solving the above two equations gives

$$
y_{2}^{*}\left(Q_{1}, Q_{2}\right)=\frac{\beta_{1} m_{X}}{\left(\beta_{1}-1\right)\left(1-\eta\left(2 Q_{1}+Q_{2}\right)\right) m_{I}},
$$

and $B_{2}=\left(y_{2}^{*}\right)^{-\beta_{1}} \frac{Q_{2}}{\left(\beta_{1}-1\right) m_{I}}$. 
The optimal capacity $Q_{2}$ of the second investment is determined by solving the following optimization problem

$$
\max _{Q_{2} \geq 0} \frac{y\left(Q_{1}+Q_{2}\right)\left(1-\eta\left(Q_{1}+Q_{2}\right)\right)}{m_{X}}-\frac{Q_{2}}{m_{I}} .
$$

The first order condition of (A48) is

$$
\frac{y\left(1-2 \eta\left(Q_{1}+Q_{2}\right)\right)}{m_{X}}-\frac{1}{m_{I}}=0,
$$

which gives

$$
Q_{2}^{*}\left(y, Q_{1}\right)=\frac{1}{2 \eta}\left(1-2 \eta Q_{1}-\frac{m_{X}}{y m_{I}}\right) .
$$

Combining (A47) and (A50) leads to the Eqs. (55) and (56).

When the firm's capacity increases from 0 to $Q_{1}$, the firm's value at the moment of first investment is equal to

$$
g_{1}\left(y, Q_{1}\right)=\frac{y Q_{1}\left(1-\eta Q_{1}\right)}{m_{X}}-\frac{Q_{1}}{m_{I}}+B_{2} y^{\beta_{1}} .
$$

Before the first investment, the value of the firm is given by

$$
v_{1}(X)=B_{1} y^{\beta_{1}}
$$

The value matching and smooth pasting conditions leads to the following equations

$$
\begin{aligned}
& B_{1} y_{1}^{* \beta_{1}}=\frac{y_{1}^{*} Q_{1}\left(1-\eta Q_{1}\right)}{m_{X}}-\frac{Q_{1}}{m_{I}}+B_{2} y_{1}^{* \beta_{1}}, \\
& \beta_{1} B_{1} y_{1}^{* \beta_{1}-1}=\frac{Q_{1}\left(1-\eta Q_{1}\right)}{m_{X}}+\beta_{1} B_{2} y_{1}^{* \beta_{1}-1},
\end{aligned}
$$

Solving these equations gives

$$
\begin{aligned}
& y_{1}^{*}=\frac{\beta_{1}}{\beta_{1}-1} \frac{m_{X}}{\left(1-\eta Q_{1}\right) m_{I}}, \\
& B_{1}=B_{2}+\frac{\left(y_{1}^{*}\right)^{1-\beta_{1}}}{\beta_{1}} \frac{Q_{1}\left(1-\eta Q_{1}\right)}{m_{X}} .
\end{aligned}
$$

The optimal capacity $Q_{1}$ of the first investment can be obtained by maximizing the firm's value $g_{1}\left(y, Q_{1}\right)$,

$$
\max _{Q_{1} \geq 0} \frac{y Q_{1}\left(1-\eta Q_{1}\right)}{m_{X}}-\frac{Q_{1}}{m_{I}}+B_{2}\left(Q_{1}\right) y^{\beta_{1}} .
$$

The first order condition is

$$
\frac{y\left(1-2 \eta Q_{1}\right)}{m_{X}}-\frac{1}{m_{I}}+\frac{\partial B_{2}\left(Q_{1}\right)}{\partial Q_{1}} y^{\beta_{1}}=0,
$$


where

$$
\begin{aligned}
B_{2}\left(Q_{1}\right) & =\left(y_{2}^{*}\left(Q_{1}\right)\right)^{-\beta_{1}} \frac{Q_{2}}{\left(\beta_{1}-1\right) m_{I}}=\frac{1-2 \eta Q_{1}\left(y_{2}^{*}\left(Q_{1}\right)\right)^{-\beta_{1}}}{\left(\beta_{1}+1\right)\left(\beta_{1}-1\right) m_{I} \eta} \\
& =\frac{1-2 \eta Q_{1}}{\left(\beta_{1}+1\right)\left(\beta_{1}-1\right) m_{I} \eta}\left(\frac{\left(\beta_{1}+1\right) m_{X}}{\left(\beta_{1}-1\right)\left(1-2 \eta Q_{1}\right) m_{I}}\right)^{-\beta_{1}} \\
& =\frac{1}{\left(\beta_{1}+1\right)\left(\beta_{1}-1\right) m_{I} \eta}\left(\frac{\left(\beta_{1}+1\right) m_{X}}{\left(\beta_{1}-1\right) m_{I}}\right)^{-\beta_{1}}\left(1-2 \eta Q_{1}\right)^{\beta_{1}+1} \\
\frac{\partial A_{2}\left(Q_{1}\right)}{\partial Q_{1}} & =\frac{1}{\left(\beta_{1}+1\right)\left(\beta_{1}-1\right) m_{I} \eta}\left(\frac{\left(\beta_{1}+1\right) m_{X}}{\left(\beta_{1}-1\right) m_{I}}\right)^{-\beta_{1}}\left(\beta_{1}+1\right)\left(1-2 \eta Q_{1}\right)^{\beta_{1}}(-2 \eta) \\
& =-\frac{2}{\left(\beta_{1}-1\right) m_{I}}\left(\frac{\left(\beta_{1}+1\right) m_{X}}{\left(\beta_{1}-1\right)\left(1-2 \eta Q_{1}\right) m_{I}}\right)^{-\beta_{1}}=-\frac{2\left(y_{2}^{*}\left(Q_{1}\right)\right)^{-\beta_{1}}}{\left(\beta_{1}-1\right) m_{I}} .
\end{aligned}
$$

Substitution of (A60) into (A58) gives

$$
\frac{y\left(1-2 \eta Q_{1}\right)}{m_{X}}-\frac{1}{m_{I}}-\frac{2}{\left(\beta_{1}-1\right) m_{I}}\left(\frac{y}{y_{2}^{*}\left(Q_{1}\right)}\right)^{\beta_{1}}=0 .
$$

Finally, substituting (A58) and (A55) into (A61) gives Eq. (53).

\section{References}

1. Dixit, A.K., Pindyck, R.S.: Investment Under Uncertainty. Princeton University Press, Princeton (1994)

2. Murto, P.: Timing of investment under technological and revenue-related uncertainties. J. Econ. Dyn. Control 31(5), 1473-1497 (2007)

3. Pennings, E., Sereno, L.: Evaluating pharmaceutical R\&D under technical and economic uncertainty. Eur. J. Oper. Res. 212(2), 374-385 (2011)

4. Nunes, C., Pimentel, R.: Analytical solution for an investment problem under uncertainties with shocks. Eur. J. Oper. Res. 259(3), 1054-1063 (2017)

5. Smets, F. Essays on Foreign direct investment. Ph.D. Thesis, Yale University, New Haven (1993)

6. Nielsen, M.J.: Competition and irreversible investments. Int. J. Ind. Organ. 20(5), 731-743 (2002)

7. Weeds, H.: Strategic delay in a real options model of R\&D competition. Rev. Econ. Stud. 69(3), 729-747 (2002)

8. Huisman, K., Kort, P.: Strategic technology adoption taking into account future technological improvement: a real options approach. Eur. J. Oper. Res. 159(3), 705-728 (2004)

9. Siddiqui, A., Takashima, R.: Capacity switching options under rivalry and uncertainty. Eur. J. Oper. Res. 222(3), 583-595 (2012)

10. Azevedo, A., Paxson, D.: Developing real option game models. Eur. J. Oper. Res. 237(3), 909-920 (2014)

11. Huisman, K.J., Kort, P.M.: Strategic capacity investment under uncertainty. RAND J. Econ. 46(2), 376408 (2015)

12. Lavrutich, M.N., Huisman, K.J.M., Kort, P.M.: Entry deterrence and hidden competition. J. Econ. Dyn. Control 69, 409-435 (2016)

13. Lavrutich, M.N.: Capacity choice under uncertainty in a duopoly with endogenous exit. Eur. J. Oper. Res. 258(3), 1033-1053 (2017)

14. Huberts, N.F.D., Dawid, H., Huisman, K., Kort, P.M.: Entry deterrence by timing rather than overinvestment in a strategic real options framework. Eur. J. Oper. Res. 274(1), 165-185 (2019)

15. Mason, C.F., Wilmot, N.A.: Jump processes in natural gas markets. Energy Econ. 46(1), 69-79 (2014)

16. McDonald, R., Siegel, D.: The value of waiting to invest. Quart. J. Econ. 101(4), $707-727$ (1986)

17. Liang, J., Yang, M., Jiang, L.: A closed-form solution for the exercise strategy in a real options model with a jump-diffusion process. SIAM J. Appl. Math. 73(1), 549-571 (2013)

18. Nunes, C., Oliveira, C., Pimentel, R.: Quasi-analytical solution of an investment problem with decreasing investment cost due to technological innovations. J. Econ. Dyn. Control. (2021). https://doi.org/10.1016/ j.jedc. 2021.104154 
19. Boyarchenko, S.: Irreversible decisions and record-setting news principles. Am. Econ. Res. 9(4), 568-577 (2004)

20. Merton, R.: Option pricing when the underlying stock returns are discontinuous. J. Financ. Econ. 3, 125-144 (1976)

21. Kou, S.G.: A jump-diffusion model for option pricing. Manag. Sci. 48, 1086-1101 (2002)

22. Kou, S.G., Wang, H.: Option pricing under a double exponential jump diffusion model. Manag. Sci. 50, 1178-1192 (2004)

23. Yan, G., Hanson, F. B. Option pricing for a stochastic-volatility jump-diffusion model with log-uniform jump-amplitudes. In: 2006 American Control Conference (2006)

24. Øksendal, B., Sulem, A.: Applied Stochastic Control of Jump Diffusions. Springer, New York (2007)

25. Pimentel, P., Nunes, C., Couto, G.: High-speed rail transport valuation with stochastic demand and investment cost. Transportmetrica A Transp. Sci. 14(4), 275-291 (2018)

26. Westman, J.J., Hanson, F.B.: Non-linear state dynamics: computational methods and manufacturing application. Int. J. Control 73(6), 464-480 (2000)

27. Fudenberg, D., Tirole, J.: Preemption and rent equalization in the adoption of new technology. Rev. Econ. Stud. 52(3), 383-401 (1985)

28. Luo, P., Tian, Y., Yang, Z.: Real option duopolies with quasi-hyperbolic discounting. J. Econ. Dyn. Control. 111, 1-12 (2020)

29. Pawlina, G., Kort, P.: Real options in an asymmetric duopoly: who benefits from your competitive disadvantage? J. Econ. Manag. Strategy 15, 1-35 (2006)

30. Thijssen, J.J., Huisman, K.J., Kort, P.M.: Symmetric equilibrium strategies in game theoretic real option models. J. Math. Econ. 48(4), 219-225 (2012)

31. Shibata, T.: Strategic entry in a triopoly market of firms with asymmetric cost structures. Eur. J. Oper. Res. 249(1), 728-739 (2016)

Publisher's Note Springer Nature remains neutral with regard to jurisdictional claims in published maps and institutional affiliations. 